\title{
Generic Platform to Generate Educational Components
}

\author{
${ }^{1}$ Yassine Aarab, Noura Aknin, ${ }^{2}$ Abdelhamid Benkaddour \\ ${ }^{1}$ Information Technology and Modeling Systems Research Unit \\ Faculty of Science,Abdelmalek Essadi University \\ Tetuan, Morocco \\ ${ }^{2}$ Computer Science, Operational Research and Applied Statistics Laboratory \\ Faculty of Science, Abdelmalek Essadi University \\ Tetuan, Morocco \\ yacinorock@gmail.com, aknin@ieee.com; ham.benkaddour@yahoo.fr
}

\begin{abstract}
In this research, we will present all the concepts necessary for the implementation of a warehouse of educational components. We define three classes of educational components: Learning components, services components and technical components. We built a metadata schema to describe them. We will study the warehouse architecture and how the actors interact with it. We will also see the features implemented at the indexing and search system that integrates. The aim is to clarify the strategic solutions we have adopted to create a generic platform, adaptable and scalable. The instantiation of this platform provides the Educational Component Repository.
\end{abstract}

Keywords: E-Learning, Component Software, Metadata, Warehouse.

\section{Introduction}

A training curriculum well built is realized by the composition of educational entities of granularity varied. In the literature, these entities are designated, sometimes with confusion, by the terms components, resources, objects or materials. Based on the definitions the more widespread, we want to propose the rules of distinction between these different terms.

Bourda uses the term educational object defined as "any entity, Digital or not, which can be used, re-used or referenced in any activity related to teaching or the learning" [1][2]such as transparencies, course notes, web pages, of simulation software, etc.

The definition more recovery by the Community has been given by the Sub-group P1484.12, belonging to the group of work (LTSC Learning Technology Standards Committee) of the IEEE responsible for describing the educational objects. This group defines a educational object, as "an entity on IT support or not, which can be used, re-used or referenced during the activities of computer-assisted learning (education intelligent- assisted by computer, learning environments, interactive distance education system, collaborative learning environments.)" [3].

It postulates that everything that can be used in the teaching by computer is considered as an educational object. A course digital, a QCM, a book are educational objects if they are used or referenced in a computerized course. By contrast, transparencies or exercises scanned, used in a traditional course, are 
not considered as educational objects because they are not included in a training curriculum computerized.

Of these definitions, one sees that an educational object must make a comprehensive information and consistent. It can thus, as mentioned Yolaine Bourda [2], establish some characteristics of an educational object to know:

\section{The autonomy}

\section{The reusability}

\section{The aggregation}

\section{The indexing}

The term educational resource is used by Grandbastien [4]. This definition is very close of the definitions of the educational object.

The term component is used by Duitama [5] with similar definitions to the concept of educational object. The term component is generally used to designate everything that can return to the constitution of a training curriculum. It is therefore usually a catch-all term that we want to reserve to the concept of software component.

We propose the use of the term:

Educational resource for designate any numeric element containing a information, even partial, intended for teaching and likely to intervene in the constitution of a course, regardless of its physical size and its digital format. For example, an image, a schema or a demonstration of the theorems are educational resources. The "Assets" in the SCORM embody the concept that we join the term of educational resource. Everything that constitutes an interest in the reuse and can be used in isolation in training is an educational resource, because its recreating requires an investment on the part of users.

Educational Object to designate any unit of content consists of a set of objects and/or teaching resources. It has a pedagogical objective, an educational sense and a duration of use necessary to its assimilation. The SCO and the aggregates in the SCORM embody the concept that we join the term of educational object. It is equivalent to the English acronym LO (Learning Object). One or more learning objects can form a course and be reused in other courses. For example, a set of web pages with courses, exercises and demonstrations constitutes an educational object.

Educational component for designer any computer application can be integrated in a training curriculum and having disciplinary content. It is the case of some Java applets or software components that we find in warehouses of educational resources. In a training curriculum, a pedagogical component can be, according to the case, considered as an educational resource or educational object. The reverse is not always true. An educational object or an educational resource is considered a component pedagogical than if it includes instructions for treatment. Of this fact, a course HTML in line is not a pedagogical component while an applet of simulation of an experiment in physics is.

There is no border well defined between these three concepts. It must rely on the experience and the personal appreciation to determine whether an entity is a pedagogical resource or an educational object. By contrast, for whether it is a component of teaching, it is wise to ask the following question "the entity owns-t-it of processing instructions at the origin of a functional process executable on the processor?". 
Yassine Aarab, Noura Aknin, Abdelhamid Benkaddour. Generic Platform to Generate Educational Components. Transactions on Machine Learning and Artificial Intelligence, Vol 5 No 4 August (2017); p: 628-636

\section{The Classes of Component Software}

\subsection{Educational Component Software}

Component Educational Software (ECS) is a business component providing a pedagogical value. It participates in the process of human learning by ensuring educational features. Typical examples are the managers of pedagogical scenarios, simulators, the resolvers problems to pedagogical vocation or the tools for evaluation of the learner.

This notion of "business component" is consistent with the Approach software engineering led by MDA models (Model Driven Architecture) which advocates to separate the aspects the trades of technical aspects during the construction of an application.

From the point of view of software engineering, the class of the ECS is well a vertical layer containing components, qualified as vertical, dedicated to a type of specific applications and therefore necessarily used by a smaller number of developers. The class of ECS corresponds to the concept of component educational software used by Laforcade [6] and appointed by the Anglophones Educational Software Component - ESC.

\subsection{Service Component Software}

Service component software (SCS) is a business component providing a functional value devoid of pedagogy. It is helpful but not necessary, to ensure the pedagogical vocation of the platform. It offers a better ergonomics and manoeuvrability of the environment.

We classify in SCS two categories of components. First, the components belonging to other vertical layers (communication, medicine, military, statistics, etc.) that have been integrated in the platforms to provide services related to their field. These are some of the components belonging to other vertical layers parallel to the class of the ECS. Second, the components used by a restricted set of areas with needs convergent.

\subsection{Technical Component Software}

Technical component software (TCS) is a component providing the basic mechanisms necessary for the proper functioning of the CES and SCS. It is a component devoid of pedagogy and features of specialty. It is likely to be used in all areas.

In e-learning, they are mainly used for the construction of the infrastructure of platforms or as intermediaries between ECS and SCS little interoperable. This is the case, for example, of components of Persistence (backup of data), printing, formatting of text (transformation of an XML text in pdf), adapter of data structures playing the role of "glue" between components, etc.

\section{Schema Dedicated to Software Component Metadata}

In the face of the absence of metadata schema dedicated to components software and more specifically to those intended for teaching and learning, we have defined a schema to meet this need. For this, we have established a work plan with the following three phases:

A first phase during which we have, in a list, compiled and classified the elements turning around this problem in the models of existing metadata. 
In a second phase, we have, but without reference to the previous study, prepared a list of useful metadata for the description of software components by adopting different points of view. (Designer, developer, assembler, user of software component) and by asking ourselves each time the following questions: "What would I like to know about this program or component? ", "What can I say about this program or component? ". The result was a list of various properties.

In a third phase, we have confronted these two lists. We have refined the organization of elements of our list, deleted the unnecessary elements and added the missing. This list has been validated by researchers of different disciplines (computer scientists, educationalists, educators).

The following diagram "Figure. 1" illustrates formally our remarks:

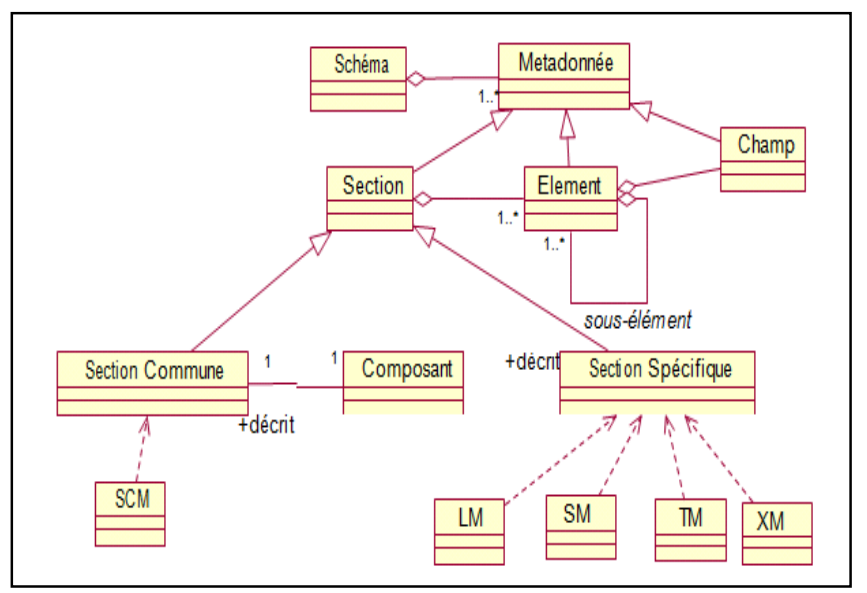

Figure1. Relationship component and sections of the diagram

Notes: The components not belonging to a domain of application can be described only with the software components Metadata (SCM) section. For the components belonging to several areas of application, we can adopt two strategies of Description:

Either, it is estimated that a domain is dominant in relation to the other, in terms of services offered and implemented by the component, and in this case, it is described with the metadata of the section specific to the field dominant.

Either one considers that the areas have the same level of dominance and in this case, it may describe the component with the sections specific to the areas dominant.

The choice of the strategy is left to the owner of the component. [7]

\subsection{Result: The Metadata of the SCM}

A schema is an entity composed of a set of metadata. At the internal level, each section contains a set of metadata hierarchically structured in the form of a tree. The nodes of the tree are called elements or subelements. They are used to organize the metadata. The leaves of the tree are called fields.

They are used to contain information. The sections, the elements and the fields are all metadata. These metadata are classified under a root element named SCM (see Fig. 2): 


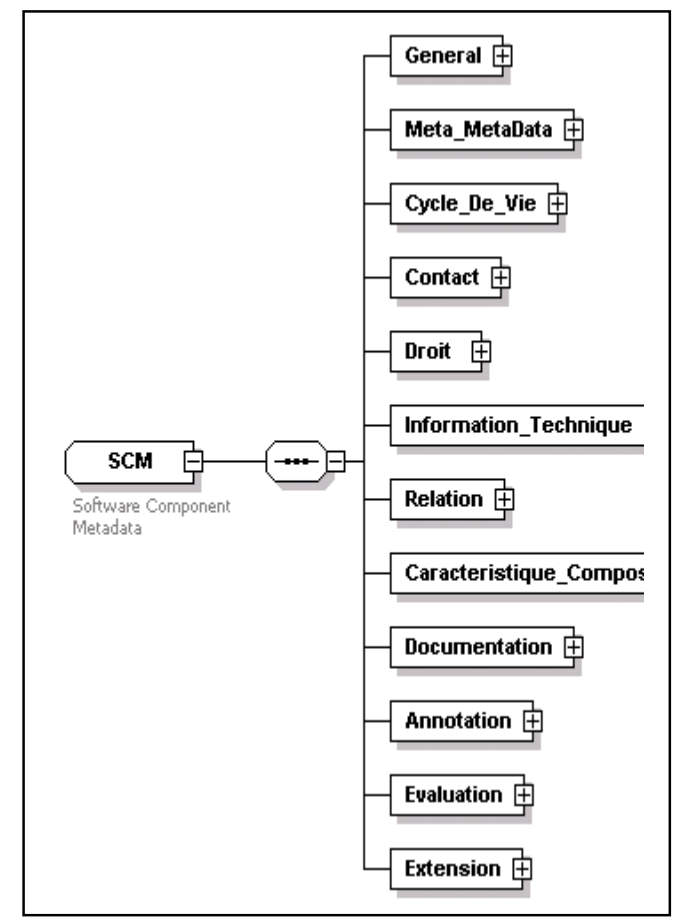

Figure2. The structure of the section "SCM"

The metadata of the SCM section allow you to describe all the software components independently of the field and the discipline. We have organized in twelve headings. These topics are for a share resumption of categories of metadata schemas as LOM and for another part of the specific additions to the needs of indexing, research and reuse of software component.

The metadata are used to find the components, they must be attached. That is why we propose a format of the package for the exchange and sharing of software components. We present, subsequently, the approach to follow to build this package and we will provide a prototype of an Intelligence Tool of metadata and generation of package.

\section{Educational Component Repository}

Before constructing the Educational Component Repository (ECR) commonly called warehouse, it is necessary to think about the features that users are entitled to expect from such a product and the best strategy to design in order to make a scalable, adaptable and generic [8].

\subsection{The features of the ECR}

The warehouse contains the software components developed by the Community e-learning. Each package of component is formed of a body and a description. The body is composed of binary code executable, the design model, source code, set of tests, documentation, etc. The description contains the metadata defined in the Fig. 3.

This fact the warehouse is composed of two parts: a catalog and a set of software components. The catalog includes the metadata of the components removed and especially information on their location. The components are archived in a file system. 


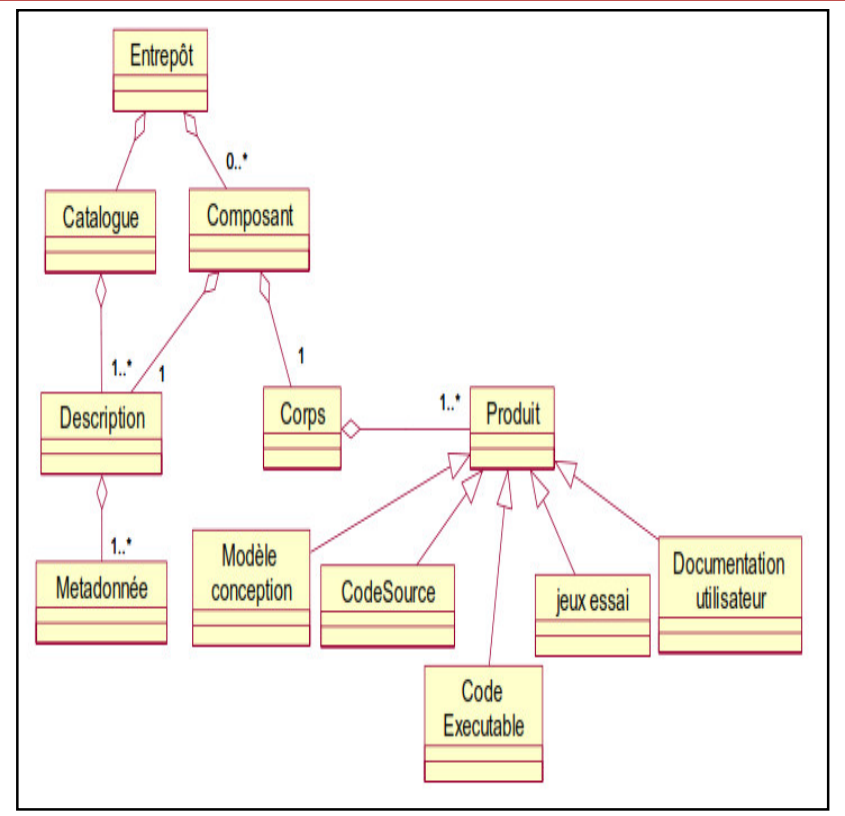

Figure3. Components of a warehouse

Identification and description of component software.

This information comes from the metadata of SCM and are recorded in the catalog.

Publication of software entities allowing developers to insert in the warehouse a new software component.

Route of the catalog allowing users to consult the description of the components software deposited in the warehouse.

Recovery of component software that allows users to obtain in their work environment a copy of the body of software component sought.

Classification and Search. In the case of a large warehouse, the course of sequential catalog is not enough. It must have the features of text search, by keyword, by attributes and by thesaurus.

For the manager of the warehouse the following features:

Storage and tracing information to use components.

Recording data on the number of access and retrieval of components, the number of successful research or not successful.

Control of access to the features offered and information contained in the warehouse.

Notification of changes to allow all potential user to be informed of the provision of a new component or an update.

\subsection{The ECR an instance of a generic platform}

The ECR should be scalable, adaptable and generic. These commitments has for consequence to complicate its design and realization because it must find a conceptual model of the platform allowing to have a independence between the metadata schema and the constituents of the warehouse: the model of the base of the index, the forms to fill used for the description and the indexing of software 
components. In other words, the platform must be totally independent of the metadata that it will manage. It is in this sense that we are talking about generic platform.

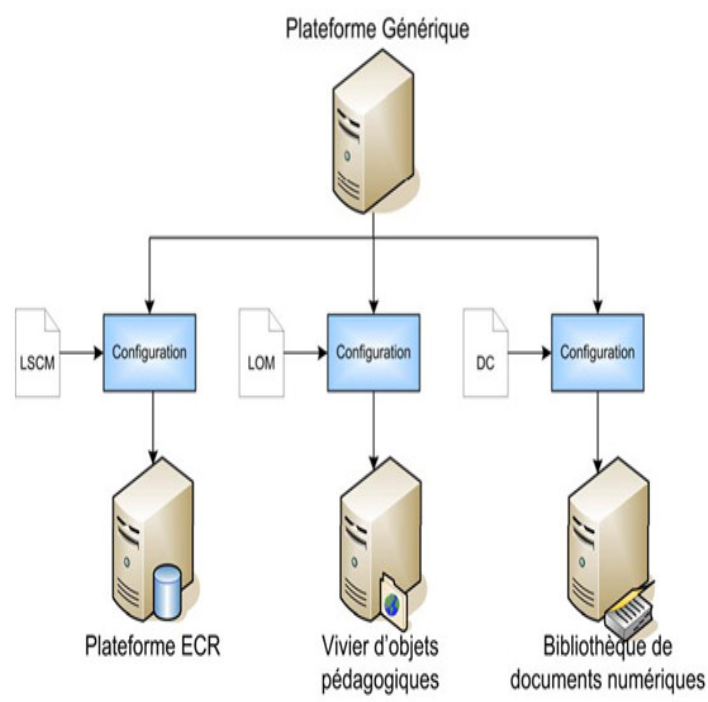

Ensures the generic nature of the Platform implies that:

The basis of the index and the XML database are not formatted

The forms of interaction are not defined

The platform includes configuration tools in order to instantiate with the properties of the models of documents to be indexed.

\subsection{Architecture of the ECR}

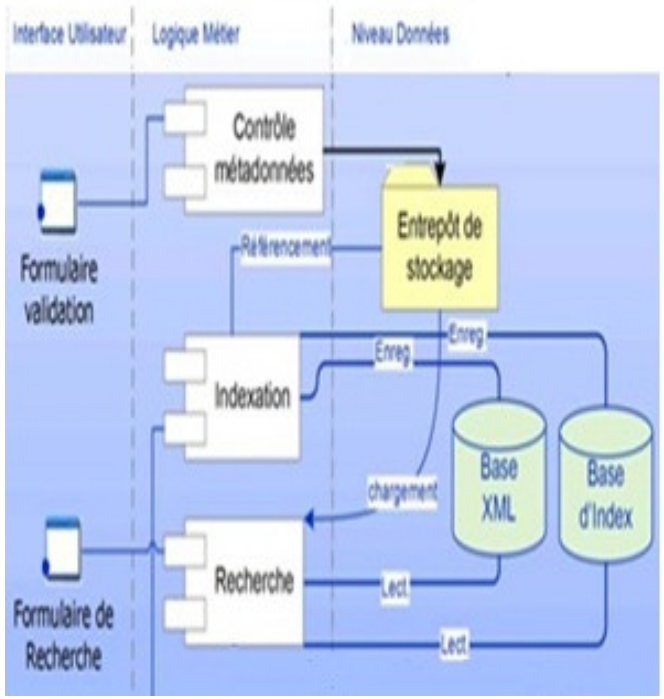

Figure5. Platform ECR (Educaional ComponentRepository) 
The ECR is a web platform developed in the form of components, respecting the MVC architecture. The most important are the component configuration, the component of indexing and the component of research. The complexity of the system and the need to control the technologies used for its development do not allow to detail here the operation of its components. In this part, the presentation will be limited to the description of the broad lines of the process "index" and "Search". Fig. 5 shows the internal architecture of the platform and the connections linking its constituents.

\subsubsection{Indexation of the components of the package}

As soon as the transfer of the package in the storage warehouse, the metadata files are extracted and transmitted to the indexing tool. They are then stored in the XML database and indexed. The basis of the index is supplied by the result of the indexation. The package, him, is archived in a folder deposit. A message is then sent to the supplier to inform him that it is become the depositary of the package and its components. From this moment, the components can be searched via the search tool integrated into the platform.

\subsubsection{Search for components in the warehouse}

To perform a search of components, the user selects the search profile the more appropriate to these criteria. A research profile essentially defines the fields on which are carried out the research and the mode used. In the case of the ECR for example, it is possible to have a form of research intended to computer specialists, educationalists, teachers, etc. searches are carried out from the base of the index. On receipt of the search query, the system provides a list of components that meet the query criteria. The user then consults with the metadata of the components of interest. If it decides to retrieve a component, the package the container will be transmitted to him in full. The search in the database of the index is done according to two additional modes: the search by keyword (with the possibility of a multicriteria research) and the search by navigation.

In the search by keyword, we have similar features to the search engines on the Internet (genus Google). As far as we are concerned, we use internally the search engine full-text Lucene of Apache [9] which allows us to have a notion of relevance in the returned documents. The XML query language that we have created provides a result in an understandable syntax by Lucene.

In the search by navigation, there is the problem of the classification of the entity according to several criteria according to the expectations of the user. In effect, a complex entity, such as component software, is quite difficult to classify according to a single hierarchical tree because it can be viewed in different ways. To respond to this difficulty of classification of results (complex entities), we use the concept of classification by facet (Prieto-Diaz, 1991) [10]. This consists of a collection of "small" lists or hierarchies of orthogonal classification to each other.

\section{Conclusion}

This work has allowed us to see that designing a generic platform is a considerable work; both on the conceptual plan that on the plan of the computing development. For that this work has a chance to continue, it is essential that this platform is entirely independent of the diagram of metadata that it uses. "Entirely" means that all the useful data of the configuration (diagram of metadata, fields of research, structure of the basis of the index and the XML base) are external to the platform, all interfaces users (to 
Yassine Aarab, Noura Aknin, Abdelhamid Benkaddour. Generic Platform to Generate Educational Components. Transactions on Machine Learning and Artificial Intelligence, Vol 5 No 4 August (2017); p: 628-636

index and search for components) are automatically generated by the platform. As well, it is possible to produce different instantiations of the platform.

\section{REFERENCES}

[1] Bourda Y. and Hélier M. (2000). Métadonnées et XML : Applications aux "objets pédagogiques". Actes TICE 2000 - Technologies of information and communication in education for engineering and industry pages 135-141.

[2] Bourda Y. (2001). Objets pédagogiques, vous avez dit objets pédagogiques ? Cahier GUTenberg, n³9-40 pages 71-79.

[3] http://Itsc.ieee.org/wg12/files/LOM_1484_12_1_v1_Final_Draft.pdf

[4] Grandbastien M. (2002). Quelques questions à propos de l'indexation et de la recherche de ressources pédagogiques sur le Web. Les technologies en éducation : Perspectives de recherche et questions vives pages 211-220.

[5] Duitama J.-F. (2005). Un modèle de composant éducatifs pour des cours adaptatifs sur le Web. France, Institut National des Télécommunication.

[6] Laforcade P. (2004). Méta-modélisation UML pour la conception et la mise en oeuvre de situationsproblèmes coopératives, Université de Pau et des pays de l'Adour.

[7] AARAB Y, AKNIN N, and BENKADDOUR H (2016). Generation System of Metadata Software Component: A Proposed Architecture.Proceeding of Mediterranean Conference On Information \& Communication Technologies 2015, Vol.381 of the series Lecture Notes in Electrical Engineering , pp 313-322.

[8] AARAB Y, AKNIN N, and BENKADDOUR H (2017).Component Based Implementation of Tool e-package. International Journal of Scientific \& Engineering Research, Volume 8, Issue 1, January-2017 820 ISSN 22295518.

[9] Lucene, Apache Lucene project: http://lucene.apache.org

[10] [8] Prieto-Diaz R. (1991). Implementing Faceted Classification for Software 